\title{
THE INTERFACE PENNY-SHAPED CRACK RECONSIDERED
}

\author{
L. M. KEER $†$ and S. H. CHEN $\ddagger$ \\ Department of Civil Engineering, Northwestern University, Evanston. IL 60201. U.S.A. \\ and \\ MARIA COMNINOU§ \\ Department of Applied Mechanics and Engineering Science, The University of Michigan, Ann Arbor, \\ MI 48109. U.S.A. \\ (Communicated by I. N. SNEDDON)

\begin{abstract}
The penny-shaped crack at the interface between two bonded dissimilar media is reconsidered on the basis of recent developments on the elimination of oscillatory singularities. This is accomplished by assuming an annular frictionless contact zone at the crack circumference and reducing the problem to a Fredholm integral equation. Expressions for the strain energy, crack opening force and bond stresses are obtained and numerical results given for specific material combinations.
\end{abstract}

\section{INTRODUCTION}

CONSIDERABLE attention has been recently directed to cracks between bonded dissimilar materials. The plane elasticity solution for a crack in the interface bond between dissimilar materials was given independently by England [1] and Erdogan [2]. The solution, which was obtained by using standard complex analysis, displayed two undesirable features that are, apparently, inherent to the problem.

(a) The stress distribution at the crack tip, in addition to the square root singularity, has an oscillatory multiplying factor of the kind $\sin (\log r)$ or $\cos (\log r)$.

(b) The crack faces overlap very close to the crack tip.

In a recent investigation by Comninou [3], which also provides an extensive literature survey on the in-plane problem, the oscillatory nature of the singularities was removed by postulating a small region of frictionless contact near the crack tip. As a consequence of this postulate the singularities at the crack tip become of the square-root type, namely, a compressive singularity in the contact region and a shear singularity in the bond.

The present investigation formulates and solves the analogous problem for a penny-shaped crack at the interface between bonded dissimilar half spaces. The conventional problem for a penny-shaped crack in an interface has been solved by Lowengrub and Sneddon [4], by using Hankel transform techniques to reduce the problem to a singular integral equation. Their conclusions are essentially the same as those of England and Erdogan for the plane elasticity problem. In the present paper techniques similar to Lowengrub and Sneddon are used to reduce the Comninou-type contact-zone problem to a singular integral equation to determine the size of the contact zone. It should be noted that the nature of the solution given by Lowengrub and Sneddon does not allow for an easy determination of the field quantities such as bond stress and crack opening displacement, but they can obtain the strain energy release rate rather easily.

FORMULATION OF PROBLEM

We consider two homogeneous, isotropic, elastic half spaces, the upper half space having elastic constants $\mu_{1}, \nu_{1}$ and the lower half space having elastic constants $\mu_{2}, \nu_{2}$, where $\mu, \nu$ denote, respectively, the shear modulus and Poisson's ratio. The two half spaces are joined together by a perfect bond everywhere, except in a region of radius $L$ (see Fig. 1) where the crack surfaces are perfectly smooth. The surface of this penny-shaped crack is subjected to a

$\nmid$ Professor.

\$raduate Student.

\&Assistant Professor. 


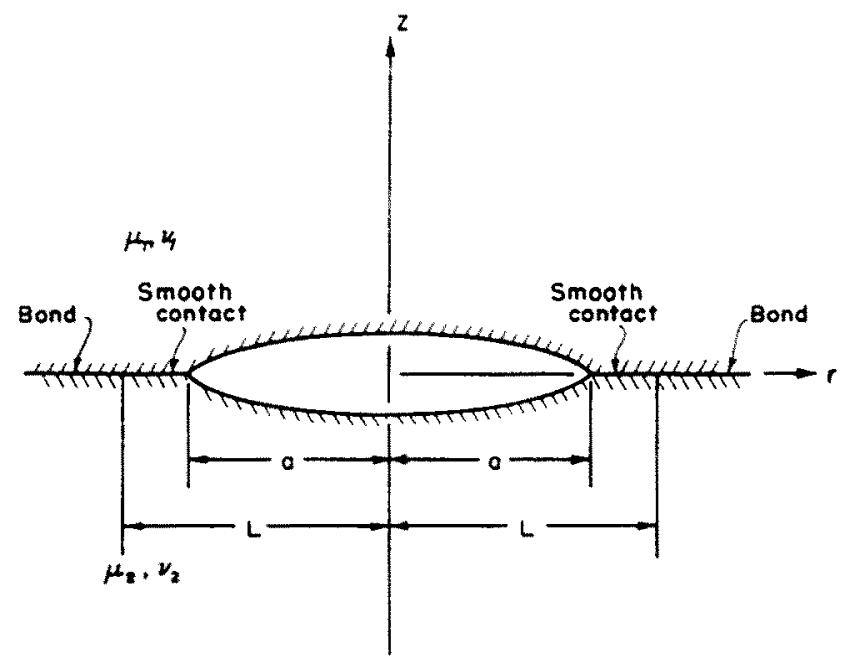

Fig. 1. Geometry and coordinate system for interfacial crack.

constant internal pressure, $p_{0}$, and it is assumed that there will be a small annular region, $a \leq r \leq L$, in which the faces of the crack make frictionless contact. Under these assumptions the boundary conditions can be written as follows

$$
\begin{aligned}
& \left.\begin{array}{l}
\sigma_{z z}^{1}=\sigma_{z z}^{2} \\
\sigma_{z r}^{1}=\sigma_{z r}^{2}
\end{array}\right\} \quad z=0,0 \leq r<\infty \\
& \left.\begin{array}{l}
\sigma_{z z}^{1}=\sigma_{z z}^{2}=-p_{0} \\
\sigma_{z r}^{1}=\sigma_{z r}^{2}=0
\end{array}\right\} z=0,0 \leq r \leq a \\
& \left.\begin{array}{c}
\sigma_{z r}^{1}=\sigma_{z r}^{2}=0 \\
u_{z}^{\prime}=u_{z}^{2}
\end{array}\right\} \quad z=0, a<r \leq L \\
& \left.\begin{array}{l}
u_{z}{ }^{1}=u_{z}^{2} \\
u_{r}{ }^{\prime}=u_{r}^{2}
\end{array}\right\} \quad z=0, L<r
\end{aligned}
$$

where superscripts 1 and 2 refer to the upper and lower half space, respectively. Equations (5) and (6) reveal the assumption made concerning the annular region of contact, $a<r \leq L$.

We introduce displacement potentials for the upper and lower half spaces in the following manner

$$
\begin{aligned}
2 \mu_{i} u_{z}^{i} & =-\partial \psi^{i} / \partial z+\left(3-4 \nu_{i}\right) \varphi^{i}-z \partial \varphi^{i} / \partial z \\
2 \mu_{i} u_{r}^{i} & =-\partial \psi^{i} / \partial r-z \partial \varphi^{i} / \partial r \\
\sigma_{z z}^{i} & =-\partial^{2} \psi^{i} / \partial z^{2}+2\left(1-\nu_{i}\right) \partial \varphi^{i} / \partial z-z \partial^{2} \varphi^{i} / \partial z^{2} \\
\sigma_{z r}^{i} & =-\partial^{2} \psi^{i} / \partial r \partial z+\left(1-2 \nu_{i}\right) \partial \varphi^{i} / \partial r-z \partial^{2} \varphi^{i} / \partial r \partial z
\end{aligned}
$$

where $\varphi^{i}, \psi^{i}, i=1,2$, must be harmonic for the equilibrium equations of elastostatics to be satisfied. The potentials are written in terms of Hankel transforms, $A_{i}(\xi), B_{i}(\xi)$, as follows

$$
\begin{aligned}
& \varphi^{\prime}=\int_{0}^{\infty} A_{1}(\xi) \mathrm{e}^{-\xi_{2}} J_{0}(\xi r) \mathrm{d} \xi \\
& \varphi^{2}=\int_{0}^{\infty} A_{2}(\xi) \mathrm{e}^{\xi_{z}} J_{0}(\xi r) \mathrm{d} \xi
\end{aligned}
$$




$$
\begin{aligned}
& \psi^{1}=-\int_{0}^{\infty} \xi^{-1} B_{1}(\xi) \mathrm{e}^{-\xi_{2}} J_{0}(\xi r) \mathrm{d} \xi \\
& \psi^{2}=\int_{0}^{\infty} \xi^{-1} B_{2}(\xi) \mathrm{e}^{\xi z} J_{0}(\xi r) \mathrm{d} \xi
\end{aligned}
$$

If eqns (9)-(16), evaluated on $z=0$, are substituted into boundary conditions (1)-(8), the latter may be written as $(i=1,2)$.

$$
\begin{array}{rlrl}
\sigma_{z z}^{j}=\int_{0}^{\infty} \xi[\beta G(\xi)+\alpha H(\xi)] J_{0}(\xi r) \mathrm{d} \xi=-p_{0} & 0 \leq r \leq a \\
\sigma_{z r}^{i}=\int_{0}^{\infty} \xi[\alpha G(\xi)+\beta H(\xi)] J_{1}(\xi r) \mathrm{d} \xi=0 & 0 \leq r \leq a \\
\sigma_{z r}^{i}=\int_{0}^{\infty} \xi[\alpha G(\xi)+\beta H(\xi)] J_{1}(\xi r) \mathrm{d} \xi=0 & a<r \leq L \\
\Delta u_{z}=\int_{0}^{\infty} G(\xi) J_{0}(\xi r) \mathrm{d} \xi=0 & a<r \leq L \\
\Delta u_{z}=\int_{0}^{\infty} G(\xi) J_{0}(\xi r) \mathrm{d} \xi=0 & L<r<\infty \\
\Delta u_{r}=\int_{0}^{\infty} H(\xi) J_{1}(\xi r) \mathrm{d} \xi=0 & L<r<\infty
\end{array}
$$

where

$$
\begin{aligned}
& \alpha=-\bar{b}\left|\left(\bar{a}^{2}-\bar{b}^{2}\right), \quad \beta=\bar{a}\right|\left(\bar{a}^{2}-\bar{b}^{2}\right) \\
& \bar{a}=\frac{\nu_{1}-1}{\mu_{1}}+\frac{\nu_{2}-1}{\mu_{2}}, \quad \bar{b}=\frac{1-2 \nu_{1}}{2 \mu_{1}}-\frac{1-2 \nu_{2}}{2 \mu_{2}}
\end{aligned}
$$

and $\Delta u=u^{1}-u^{2}$. Note that $\alpha, \beta$ have the relationship, $\alpha=\hat{\beta} \beta$ where $\hat{\beta}$ is Dundurs constant [5], i.e.

$$
\hat{\beta}=\frac{\mu_{2}\left(\kappa_{1}-1\right)-\mu_{1}\left(\kappa_{2}-1\right)}{\mu_{2}\left(\kappa_{1}+1\right)+\mu_{1}\left(\kappa_{2}+1\right)}
$$

where $\kappa_{i}=3-4 \nu_{i}, i=1,2$.

To solve the coupled set of integral eqns (17)-(22), it is convenient to introduce the following finite Fourier sine and cosine transforms as follows

$$
G(\xi)=\int_{0}^{a} \varphi(t) \sin (\xi t) \mathrm{d} t, H(\xi)=\int_{0}^{L} \psi(t) \cos (\xi t) \mathrm{d} t
$$

where the choice of $G(\xi)$ will automatically satisfy eqns (20) and (21), and where to avoid a jump in the radial displacement in $L<r<\infty$, and to satisfy eqn (22), it is required that

$$
\int_{0}^{L} \psi(t) \mathrm{d} t=0
$$

From the definition of the transforms, eqns (28) and (29), and the symmetry of the problem, $\psi(t)$ must be an even function and, therefore

$$
\int_{-L}^{L} \psi(t) d t=0
$$


Let us now integrate eqns (18) and (19) to obtain

$$
\int_{0}^{\infty}[\alpha G(\xi)+\beta H(\xi)] J_{0}(\xi r) \mathrm{d} \xi=C \quad 0 \leq r \leq L
$$

where $C$ is a constant of integration. Upon multiplying eqn (32) by the operator $\int_{0}^{x} r\left(x^{2}-r^{2}\right)^{-1 / 2}$ $\mathrm{d} r$ and reversing the order of integration, we obtain

$$
\frac{\alpha}{2} \int_{0}^{a} \varphi(t) \log \frac{x+t}{x-t} \mathrm{~d} t+\frac{\pi}{2} \beta \int_{0}^{x} \psi(t) \mathrm{d} t=C x
$$

Differentiating (33) leads to the following singular integral equation

$$
\frac{\alpha}{2} \int_{-a}^{a} \frac{\varphi(t) \mathrm{d} t}{t-x}+\frac{\pi}{2} \beta \psi(x)=C \quad 0 \leq x \leq L .
$$

In a similar manner eqn (17) can be reduced to

$$
\frac{\pi}{2} \beta \varphi(x)-\frac{\alpha}{2} \int_{-1}^{t} \frac{\psi(t) \mathrm{d} t}{t-x}=-p_{0} x \quad 0 \leq x \leq a .
$$

The singular integral eqns (34) and (35) can be put into a single integral equation by using a method similar to that of Comninou [3]. Express $\psi$ in (34) in terms of $\varphi$ and then substitute into (35), which leads to

$$
\frac{\pi}{2} \beta \varphi(r)-\frac{\alpha}{\pi \beta} C \oint_{-L}^{L} \frac{\mathrm{d} t}{t-r}+\frac{\alpha^{2}}{2 \pi \beta} \int_{-a}^{a} \varphi\left(t^{\prime}\right) \mathrm{d} t^{\prime} \int_{-L}^{L} \frac{\mathrm{d} t}{(t-r)\left(t^{\prime}-t\right)}-\frac{\alpha^{2} \pi}{2 \beta} \varphi(r)=-p_{o} r
$$

where the last term arises from the application of the Poincaré-Bertrand formula. Since

$$
\frac{1}{(t-r)\left(t^{\prime}-t\right)}=\frac{1}{t^{\prime}-r}\left(\frac{1}{t-r}+\frac{1}{t^{\prime}-t}\right)
$$

eqn (36) can be further simplified to

$$
\frac{\pi}{2}\left(1-\hat{\beta}^{2}\right) \varphi(r)-\frac{\hat{\beta} C}{\pi \beta} \log \left(\frac{L-r}{L+r}\right)+\frac{\hat{\beta}^{2}}{2 \pi} \int_{-a}^{a} \frac{\varphi(t)}{t-r} \cdot \log \left[\frac{(L-r)(L+t)}{(L-t)(L+r)}\right] \mathrm{d} t=-\frac{p_{0} r}{\beta} \quad 0<r<a .
$$

The constant $C$ in eqn (37) can be obtained by integrating (34) and using eqn (31), and it is given as

$$
C=-\frac{\alpha}{4 L} \int_{-a}^{a} \varphi(t) \log \frac{L-t}{L+t} \mathrm{~d} t
$$

Equation (37) can be put into nondimensionalized form by the substitutions

$$
r=a \zeta, t=a w, \gamma=a / L, \varphi(t)=\frac{p_{0} a}{\beta} \Phi(w)
$$

giving the Fredholm integral equation

$$
\left(1-\hat{\beta}^{2}\right) \Phi(\zeta)+\frac{\hat{\beta}^{2}}{\pi^{2}} \int_{-1}^{1} \Phi(w) l(\zeta, w) \mathrm{d} w=-\frac{2}{\pi} \zeta
$$


where

$$
\begin{aligned}
& l(w, \zeta)=k(w, \zeta)+\frac{\gamma}{2} \log \frac{1-\gamma \zeta}{1+\gamma \zeta} \log \frac{1-\gamma w}{1+\gamma w} \\
& k(w, \zeta)=\frac{1}{w-\zeta}\left\{\log \left[\frac{(1-\gamma \zeta)(1+\gamma w)}{(1+\gamma \zeta)(1-\gamma w)}\right]\right\} .
\end{aligned}
$$

It can be shown that $k(w, \zeta)$ has the following property

$$
k(w, \zeta) \rightarrow \frac{2 \gamma}{1-\gamma^{2} \zeta^{2}} \text { as } w \rightarrow \zeta
$$

We note that in the present form the size of the contact region, $L-a$, is not yet determined. Its size may be found from the condition that the crack must close smoothly at $r=a$, or equivalently, that the normal stress, $\sigma_{z z}$, does not exhibit a singularity at $r=a$. The normal and shear stresses on $z=0$ may be calculated from

$$
\begin{aligned}
& \sigma_{2 z}=\int_{0}^{\infty} \xi[\beta G(\xi)+\alpha H(\xi)] J_{0}(\xi r) \mathrm{d} \xi \\
& \sigma_{z r}=\int_{0}^{\infty} \xi[\alpha G(\xi)+\beta H(\xi)] J_{1}(\xi r) \mathrm{d} \xi
\end{aligned}
$$

where $G(\xi)$ and $H(\xi)$ are given by (28) and (29). After performing the necessary manipulations, the stresses are obtained as

$$
\begin{aligned}
& \sigma_{z z}=-\frac{\beta \varphi(a)}{\left(r^{2}-a^{2}\right)^{1 / 2}}+\beta \int_{0}^{a} \frac{\varphi^{\prime}(t) \mathrm{d} t}{\left(r^{2}-t^{2}\right)^{1 / 2}}+\frac{\alpha \psi(L)}{\left(L^{2}-r^{2}\right)^{1 / 2}}-\alpha \int_{r}^{L} \frac{\psi^{\prime}(t) \mathrm{d} t}{\left(t^{2}-r^{2}\right)^{1 / 2}}, \quad a<r<L \\
& \sigma_{z z}=-\frac{\beta \varphi(a)}{\left(r^{2}-a^{2}\right)^{1 / 2}}+\beta \int_{0}^{a} \frac{\varphi^{\prime}(t) \mathrm{d} t}{\left(r^{2}-t^{2}\right)^{1 / 2}} \quad L<r<\infty \\
& \sigma_{z r}=-\frac{\beta}{r} \int_{0}^{r} \frac{t \psi^{\prime}(t) \mathrm{d} t}{\left(r^{2}-t^{2}\right)^{1 / 2}} \quad a<r<L \\
& \sigma_{z r}=\frac{\beta \psi(L)}{\left(r^{2}-L^{2}\right)^{1 / 2}} \frac{L}{r}-\frac{\beta}{r} \int_{0}^{r} \frac{t \psi^{\prime}(t) \mathrm{d} t}{\left(r^{2}-t^{2}\right)^{1 / 2}}, \quad L<r<\infty .
\end{aligned}
$$

The condition that the crack must close smoothly at $r=a$ is given by $\varphi(a)=0$ (or $\Phi(1)=0)$ and can be satisfied by requiring that

$$
F=\hat{\beta}^{2} \int_{-1}^{l} \Phi(w) l(w, 1) \mathrm{d} w+2 \pi=0 .
$$

Thus, eqns (40) and (50) can be used to solve for $\Phi$ and $\gamma$. Let $\psi(t)=p_{0} a \Psi / \beta$ and with the aid of (34) it is determined from

$$
\Psi(\zeta)=-\frac{\gamma \hat{\beta}}{2 \pi} \zeta-\frac{\hat{\beta}}{\pi} \int_{-1}^{1} \frac{\Phi(\xi) \mathrm{d} \xi}{\xi-\zeta}
$$

where

$$
C=\int_{-1}^{1} \Phi(\xi) \log \frac{1-\gamma \xi}{1+\gamma \xi} \mathrm{d} \xi
$$

Physical quantities. Once eqns (40) and (50) have been solved, the important physical quantities may be determined. Of interest is the bond stress distribution for $r>L$, which may 
be found in a form suitable for numerical calculation from eqns (47) and (49) as

$$
\begin{gathered}
\sigma_{z z}(r)=p_{0} \int_{0}^{1} \frac{\Phi^{\prime}(\xi) \mathrm{d} \xi}{\left(r^{2}-\xi^{2}\right)^{1 / 2},} \quad r>\frac{1}{\gamma} \\
\sigma_{r z}(r)=p_{0}\left[\Psi\left(\frac{1}{\gamma}\right) /\left(\gamma r\left(r^{2}-\gamma^{-2}\right)^{1 / 2}\right)-\frac{1}{r} \int_{0}^{1 / \gamma} \frac{\xi \Psi^{\prime}(\xi) \mathrm{d} \xi}{\left(r^{2}-\xi^{2}\right)^{1 / 2}}\right], \quad r>\frac{1}{\gamma} .
\end{gathered}
$$

The strain energy is given by

$$
E=\pi p_{0} \int_{0}^{a}\left[u_{z}^{1}(r, 0)-u_{z}^{2}(r, 0)\right] r \mathrm{~d} r
$$

where

$$
u_{z}^{1}(r, 0)-u_{z}^{2}(r, 0)=\int_{0}^{\infty} G(\xi) J_{0}(\xi r) \mathrm{d} \xi
$$

After suitable manipulations, the strain energy is found in a relatively simple form for calculations as

$$
E=\frac{p_{0}^{2} \pi \gamma^{3} L^{3}}{\beta} \int_{0}^{1} \xi \Phi(\xi) \mathrm{d} \xi
$$

The stress intensity factor for Mode II is given as

$$
K_{2}=\lim _{r \rightarrow L^{+}}[2(r-L)]^{1 / 2} \sigma_{z r}
$$

or

$$
K_{2}=p_{0} L^{1 / 2} \gamma \Psi(1 / \gamma)
$$

We note that since the dominant singularity in the normal stress has been removed at $r=a$, there will be no stress intensity factor in Mode I, as found by Comninou. However, as can be seen by eqn (46), there is a contact stress singularity at $r=L$ that depends upon the value of $\Psi(1 / \gamma)$. This singularity is given as

$$
K_{1}=\lim _{r \rightarrow L^{-}}[2(L-r)]^{1 / 2} \sigma_{z z}
$$

or

$$
K_{1}=p_{0} L^{1 / 2} \gamma \hat{\beta} \psi(1 / \gamma)
$$

Hence,

$$
K_{1} / K_{2}=\hat{\beta}
$$

Note that if normal tractions are negative, $K_{1}<0$, then $\hat{\beta}>0, K_{2}$ must be negative. This sign differs from that of Comninou due to the choice of coordinate system in the present case.

\section{NUMERICAL SOLUTIONS AND RESULTS}

It should be noted that, in contrast to the analogous 2-dimensional problem solved by Comninou, the present analysis only requires the solution of a Fredholm integral equation rather than a singular integral equation. The numerical solution is, therefore, somewhat simplified since the collocation points and integration points can be taken as being the same. 
The method used is the following. We wish to solve eqn (40), subject to the relation given by eqn (50). Let $\Phi(\xi)=f(\xi)\left(1-\xi^{2}\right)^{-1 / 2}$ and use the Gaussian quadrature formula for Chebyshev polynomials of the first kind with roots and weights given by $\xi_{i}=\cos [(2 i-1) \pi / 2 n]$ and $w_{i}=\pi / n$, respectively, where $n$ is the number of points. Values of $\gamma$ are prescribed to determine $\Phi(\xi)$, which is then put into eqn (50). When the functional relationship of $F$ vs $\gamma$ is found, the correct value of $\gamma$ that satisfies (50) can be easily determined. The function chosen for the numerical evaluation of $\Phi(\xi)$ provided good accuracy for the numerical calculation.

The value of $\gamma=a / L$ was found to vary from $\gamma>1-10^{-7}$ for $\hat{\beta}<0.4(\gamma=0.9999932$ for $\hat{\beta}=0.4239$ ) to $\gamma=0.9998$ for $\hat{\beta}=0.5$. Thus, for all possible values of $\hat{\beta}$ the values for $\gamma$ are very close to unity although they seem slightly smaller than those found in [3].

To obtain the physical quantities it is necessary to develop a representation for the value of $\Phi(\zeta), 0<\zeta<1$. This can be easily done by representation of $f(\zeta)$ by Chebyshev polynomials (see e.g. Krenk[6]) as

$$
\begin{gathered}
f(x)=\sum_{i=0}^{n-1} c_{i} T_{j}(x) \\
c_{j}=\frac{2}{n} \sum_{i=1}^{n} \cos \left[\frac{2 i-1}{2 n} j \pi\right] f\left(x_{i}\right) \\
x_{i}=\cos \frac{2 i-1}{2 n} \pi .
\end{gathered}
$$

We note that (Erdogan, Gupta, Cook [7])

$$
\frac{1}{\pi} \int_{-1}^{1} T_{n}(t)\left(1-t^{2}\right)^{-1 / 2} \frac{\mathrm{d} t}{t-s}=\frac{\left[\left(s^{2}-1\right)^{1 / 2}-s\right]^{n}}{(-1)^{n+1}\left(s^{2}-1\right)^{1 / 2}}
$$

and thus

$$
\Psi(\zeta)=-\frac{\gamma \hat{\beta}}{2 \pi} \zeta+\hat{\beta} \sum_{j=0}^{n-1} c_{j} \frac{\left[\zeta-\left(\zeta^{2}-1\right)^{1 / 2}\right]^{\prime}}{\left(\zeta^{2}-\right)^{1 / 2}}
$$

Having calculated $\Phi, \Psi$, it is possible to compute the bond stresses from eqns (53) and (54). For $\hat{\boldsymbol{\beta}}=0.4854$ these distributions are tabulated in Table 1 . An attempt was made to compare the result with the one of Lowengrub and Sneddon [4], but as they point out the integrals in their eqn (5.6) are extremely difficult to evaluate numerically. Unlike the 2-dimensional problem [1,2], where the bond stresses have a closed-form representation, the 3-dimensional problem involves the integration of highly oscillatory integrals. For the value of $\hat{\beta}=0.4854$, the shear stress intensity factor $K_{2} / p_{0} L^{1 / 2}=-0.6772$.

The crack extension force, $G$, can be calculated in a manner similar to that of [3]. Let the change in the total free energy $\delta \mathrm{E}$, when $a$ advances to $a+\delta a$ and $L$ extends to $L+\delta L$, be given by

$$
\delta E=-\pi\left[\int_{a}^{a+\delta a} n \sigma_{z z}(r, 0) \Delta u_{z}(r, 0) \mathrm{d} r+\int_{L}^{L+\delta L} r \sigma_{r z}(r, 0) \Delta u_{r}(L+\delta L, 0) \mathrm{d} r\right]
$$

The first integral will give no contribution, and the second integral can be evaluated by noting that

$$
\sigma_{r z} \sim \frac{K_{2}}{[2(r-L)]^{1 / 2}}, \quad \Delta u_{r}(L+\delta L, 0) \sim \frac{K_{2}}{\beta}[2(r-L+\delta L)]^{1 / 2} .
$$

Thus

$$
G=-\frac{\delta E}{\delta L}=\frac{\pi^{2} K_{2}^{2}}{2 \beta} L
$$


is the crack opening force. We note that $G$ can be found from the strain energy calculation. eqn (57), as

$$
G=-\frac{\delta E}{\delta L}=\frac{3 L^{2} \gamma^{3} p_{0}^{2} \pi}{\beta} \int_{0}^{1} \xi \Phi(\xi) \mathrm{d} \xi
$$

The difference between these two calculations is about $0.69 \%$. If this value is compared with that computed by Ref. [2], the difference is about 3\%, where in the latter reference their eqn (5.5) should read

$$
W=\frac{p_{0}^{2}}{6 G_{2}}\left\{\left(1+\kappa_{1} \Gamma\right)\left(\kappa_{2}+\Gamma\right)\right\}^{1 / 2} \pi \gamma \operatorname{cosech}\left(\frac{1}{2} \pi \gamma\right)\left(1+\frac{1}{4} \gamma^{2}\right)
$$

\begin{tabular}{|c|c|c|}
\hline$r L$ & $-\sigma_{r z}(r, 0)\left(\frac{r^{2}}{L^{2}}-1\right)^{1 / 2} / p_{0}$ & $\sigma_{z z}(r, 0) / p_{0}$ \\
\hline 1.0000 & 0.6772 & 20.42 \\
\hline 1.0001 & 0.6686 & 17.00 \\
\hline 1.0002 & 0.6500 & 15.05 \\
\hline 1.0005 & 0.6098 & 11.97 \\
\hline 1.0010 & 0.5677 & 9.603 \\
\hline 1.0020 & 0.5169 & 7.423 \\
\hline 1.0050 & 0.4395 & 5.010 \\
\hline 1.0100 & 0.3753 & 3.568 \\
\hline 1.0500 & 0.2212 & 1.358 \\
\hline 1.1000 & 0.1597 & 0.8029 \\
\hline 1.2000 & 0.1066 & 0.4312 \\
\hline 1.5000 & 0.0550 & 0.1581 \\
\hline 2.0000 & 0.0309 & 0.0654 \\
\hline
\end{tabular}

For $\hat{\beta}=0.4854, E \doteq 0.1114 p_{0}^{2} L^{3}$, using eqn (57).

Acknowledgement-The work reported here was carried out in the course of research sponsored by the U.S. Army Office Durham under Grant DAAG 29-78-G-0031. The authors should like to acknowledge helpful discussions with Prof. J. D. Achenbach.

\section{REFERENCES}

[1] A. H. ENGLAND, I. Appl. Mech. 32, 400 (1965).

[2] F. ERDOGAN, J. Appl. Mech. 32, 403 (1965).

[3] M. COMNINOU, J. Appl. Mech. 44, 631 (1977).

[4] M. LOWENGRUB and I. N. SNEDDON, Int. J. Eng. Sci. 12,387 (1974)

[5] J. DUNDURS, J. Appl. Mech. 36, 650 (1969).

[6] S. KRENK, Q. Appl. Math. 32, 479 (1975).

[7] F. ERDOGAN, G. D. GUPTA and T. S. COOK, In Methods of Analysis and Solutions of Crack Problems (Edited by G. C. Sih), pp. 368-425 (1973). 